\title{
Personalized Therapeutics and Pharmacogenomics: Integral to Personalized Health Care
}

\author{
Wolfgang Sadee ' (D)
}

Received: 26 April 2017 / Accepted: 27 April 2017 / Published online: 10 May 2017

(C) Springer Science+Business Media New York 2017

This Theme Issue of Pharmaceutical Research assembles a series of original papers and reviews on personalized therapeutics and pharmacogenomics, a rapidly expanding field fostering fundamental changes in personalized health care. Stunning advances in novel technologies and disciplines propel unprecedented insights in biomedical sciences, stimulating profound changes in global health care (1-3) and converging on personalized health care (Fig. 1). Our growing ability to gather immense information on an individual's health status and genomic profile compels a shift of health care from onedrug-treats-all towards personalized medicine. Assembled in electronic health records, and increasingly available across health care systems, personal genomics holds promise for driving innovations in modern health care (3). We expect to gain a better understanding on how each individual's unique characteristics reveal resilience in health, susceptibility to disease, and response to interventions and therapy. This approach has been variably dubbed personalized medicine, personalized health care, precision medicine, and $\mathrm{P} 4$ medicine. The last, coined by Leroy Hood, invokes predictive, preventive, personalized, and participatory medicine, acknowledging the important role every patient has in his or her own health care. Recently, the term precision medicine has become popular. By invoking precision, we imply that diseases primarily result from dominant influence of one or only a few well defined key factors, such as critical mutations that can be targeted precisely. However, this is unlikely to be a common scenario, even in cancers driven by somatic mutations. While such driver mutations can be targeted 'with precision', treatments are rarely curative because of inherent underlying complexity of cancer

Wolfgang Sadee

wolfgang.sadee@osumc.edu

Center for Pharmacogenomics, Department of Cancer Biology and

Genetics, College of Medicine, The Ohio State University,

Columbus, $\mathrm{OH} 43210$, USA biology. In addition, the term 'precision medicine' detracts from recognizing the importance of complex personal, environmental, and societal factors, all key to personalized health care. Therefore, recognizing our inability to 'precisely' understand human complexity, I prefer the term personalized medicine or health care, the latter emphasizing the opportunities arising from multifaceted health maintenance, disease prevention, early intervention, and disease management.

With this background, we consider "personalized therapeutics" germane to the pharmaceutical sciences. As a result of the dramatic expansion of genomics, drug therapy is further transformed by pharmacogenomics - the use of genomics information to optimize an individual's health care and therapy. Today genomics is understood to encompass all -omics areas, including integration between diverse data on DNA, RNA, protein, metabolite, epigenome, metagenome, etc. The articles in this issue address the confluence between therapeutics and our new understanding of the relevance of interindividual differences, including genomics, highlighting the challenges we face and opportunities we can pursue.

With dramatic human population growth, drastic environmental changes, and rapid genetic adaptation emerging in parallel $(4,5)$, we face growing challenges in maintaining health, preventing disease progression, and treating disease effectively. Dense cohabitation facilitates spread of infectious disease, thereby leading to an evolutionary process that strengthens the innate immune system as a primary defense mechanism. In turn, a hyper-active innate immune system results in autoimmune and inflammatory diseases that have become increasingly prevalent (6). Rapid population growth accelerates adaptations of the human genome to changing environments, favoring genetic variants that promote reproductive success but do not necessarily protect against chronic disease. Given the diversity in genetics, environment, behaviors, and social factors, drug therapy often fails to provide benefit and even causes substantial harm. Adverse drug effects, defined by WHO definition to be unavoidable even if 


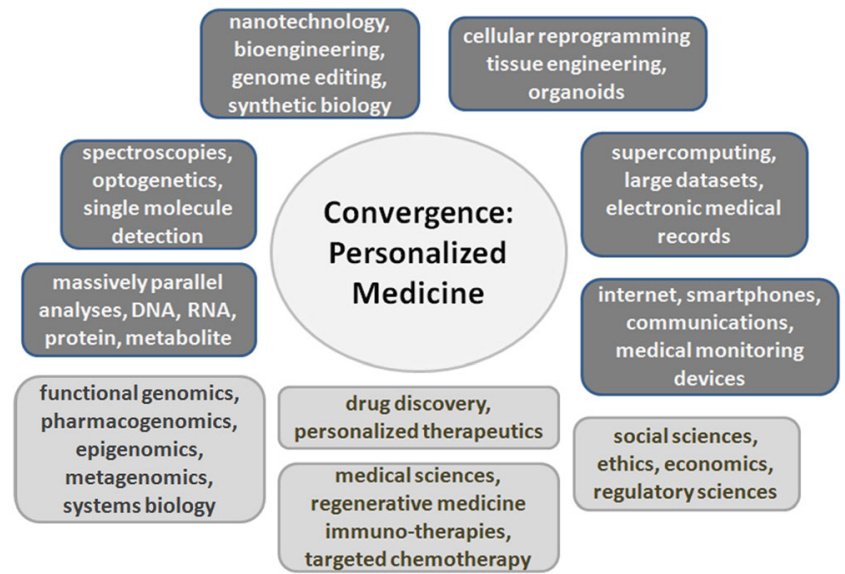

Fig. I The changing landscape of personalized medicine. Profound advances in multiple disciplines along with stunning technological innovations compel a worldwide move towards personalized medicine - a roadmap towards improved health care and therapy $(\mid-3)$

the drug is given according to standard recommendations, are considered a leading cause of morbidity and mortality (7).

It has become evident that the maintenance of health, effective disease prevention, and therapy depend on key factors that dynamically interact with each other: environment, diet, exercise, age, sex, socioeconomic status, cultural context, and access to health care. Drug therapy is only one part of the health care continuum, and by itself unlikely to be effective unless well integrated into an individual's personal life style. Diet intervention, exercise programs, and behavioral changes are all critical and often can replace drug therapy. For example, rigorous exercise might not only prevent disease and slow aging, but could also replace drug therapy under certain circumstances (8). Similarly, near universal use of smart phone devices leads to new health apps that monitor an individual's physical status and can lead to life style changes that also could obviate the need for drug therapy (9). In addition, continuous measurements of symptoms, such as blood pressure and insulin, educate drug dosage schedules. This raises questions whether and when sustained release drug formulations are indeed warranted or may be counterproductive. Glucose levels and blood pressure fluctuate, often synchronous with the circadian rhythm - now recognized as an important factor in health and therapy response (10). Recently, dosage regimen adjusted to continuous personal health measures have become feasible, leading to improved health care. Moreover, genetics and genomics can serve as an additional guidepost, in a growing number of instances already part of standard care - an endeavor still in infancy but making substantive progress.

Spectacular advances in genomic medicine have led to discovery of single genes or mutations linked to the disease process. These include driver mutations in cancer amenable to targeted chemotherapy, and CFTR mutations causing protein defects that can be corrected with suitable ligands to restore normal functions. Over the past $4-5$ years, $\sim 20 \%$ of all newly FDA-approved drugs require a companion biomarker test so that only those who benefit receive the treatment (11). Some project obligatory biomarker tests to account for up to $70 \%$ of New Drug Approvals, as blockbuster drugs treating a complex disorder broadly have proven increasingly difficult to develop. We can further anticipate gene therapy (example (12)), in vivo gene editing, including the use of CRISPR (13), and immune modulation $(14,15)$ to assume a critical role in medicine. While epigenomics focuses on chromatin and gDNA modifications that can be long lasting with profound effects on disease and drug response, a nexus between genetic variants and epigenetic marks (16) is often overlooked but highlights genetic predisposition to the influence of environmental factors. Lastly, somatic mutations that accumulate over time are thought to contribute to aging and cancer, but also could be critical to other diseases (17). If somatic mutations lead to clonal expansion, a majority of target cells could carry the mutations. For example, relatively frequent somatic mutations of TET2, which modulates the immune system by oxidizing methylated $C p G$, lead to clonal expansion of hematopoetic stem cells without causing cancer. In a mouse model, tet $2^{-/-}$macrophages secrete more IL1 $\beta$ upon stimulation and promote atherosclerotic cardiovascular disease, offering new opportunities for therapies (18). Yet, common complex disorders thus far have largely defied attempts to develop clinically compelling biomarker tests even while genetic factors (broad sense heritability) contribute a substantial portion to disease risk and treatment outcome. Managing complex diseases and health maintenance into advanced age requires integration of life-style changes and dietary programs, with drug therapies as complementary strategies. Similar developments in individualized therapies are beginning to emerge in veterinary medicine (19), and human therapeutics can benefit from those advances that can be more readily implemented in animals.

This Theme Issue of Pharmaceutical Research addresses personalized therapeutics with pharmacogenomics as a means for determining who will respond favorably or experience adverse effects when given a prescribed drug. In a systematic review, we had previously addressed the question whether genetic factors can account for adverse drug reactions, finding that the most offensive drugs tend to be metabolized by highly polymorphic enzymes such as CYP2D6 (7). Therefore, some ADRs are indeed avoidable if one considers genetic differences between individuals in prescribing drugs. Meanwhile, the FDA has assembled a table of pharmacogenomic biomarkers with $>150$ gene drug pairs of clinical relevance (https://www.fda.gov/Drugs/ScienceResearch/ ResearchAreas/Pharmacogenetics/ucm083378.htm). Nevertheless, adoption of such genetic biomarkers in the clinic has been remarkably slow, in part because any single genetic variant typically accounts for only a fraction of genetic influence on therapy outcome. Therefore, personalized 
therapeutics requires the integration of multiple genomic markers with all other factors influencing an individual's response - a feat yet to be implemented successfully.

The articles in this Theme Issue critically evaluate the influence of these interacting factors, the search for critical genetic variants, and evaluation of clinical utility, in an effort to set beacons for therapeutic advances to come. Five review articles cover a broad spectrum of topics relevant to personalized therapeutics. G.T. Tucker delivers a critical perspective on personalized medicine, assessing diverse factors influencing individualization of drug dosage (Personalized drug dosage closing the loop). M.M. Dias, M.J. Sorich, A. Rowland, M.D. Wiese, and R.A. McKinnon assess routine use of pharmacogenetic tests (The routine clinical use of pharmacogenetics tests: what will it require). Going beyond ad hoc genotyping when the need arises, K.W. Weitzel, L.H. Cavallari, and L.J. Lesko discuss prospective or preemptive use of pharmacogenetic biomarker panels that require new clinical decision tools for broad implementation. Addressing the growing clinical use of biologics, N. Vande Casteele and R. Khanna evaluate clinical drug monitoring of a protein drug and the role of antidrug antibodies (Therapeutic drug monitoring of golimumab in the treatment of ulcerative colitis). Focusing on drug induced liver toxicity, A.K Daly addresses the question whether genetic factors influencing drug disposition affect drug induced liver injury (Are polymorphisms in genes relevant to drug disposition predictors of susceptibility to drug-induced liver injury?). These reviews are followed by eight original research papers targeting specific topics. Y. Sugiyama and coworkers (T. Yoshikado et al.) report on complex drugdrug interactions, involving interactions between OATP transporters and CYP3A enzymes and drugs that serve as dual substrates for both (A clinical cassette dosing study for evaluating the contribution of hepatic OATPs and CYP3A to drugdrug interactions). K. Toshimoto, A. Tomaru, M. Hosokawa, and Y. Sugiyama assess the influence of genetic variants of enzymes and transporters on irinotecan induced side effects Virtual clinical studies to examine the probability distribution of the AUC at target tissues using physiologically-based pharmacokinetic modeling: Application to analyses of the effect of genetic polymorphism of enzymes and transporters on irinotecan induced side effects). This theme is expanded in a further paper from the same group, by $\mathrm{H}$. Kusuhara et al. on drug-transporter interactions involving OATPs that affect endogenous substrates of these transporters (Investigation of glycochenodeoxycholate sulfate and chenodeoxycholate glucuronide as surrogate endogenous probes for drug interaction studies of OATP1B1 and OATP1B3 in healthy Japanese volunteers). A clinical study by J. Luzum, J.P. Kitzmilller, et al. addresses the role of CYP2D6 null mutations on the response to beta-blocker therapy of heart failure, showing that patients with poor CYP2D6 metabolizer status fail to reach recommended metoprolol dosage regimen and may benefit from adjusting dosing targets (CYP2D6 Genetic Variation and Beta-Blocker Maintenance Dose in Patients with Heart Failure). Polymorphisms in the transmembrane regions of $B C R P$ (encoding the breast cancer resistance protein transporter) are characterized by N. Sjostedt, J.J.M.W. van den Heuvel, J.B. Koenderink, and H. Kidron, revealing significant defects in transport function (Transport Activity of ABC Transporter ABCG2). Similarly, X. Wen, M.S. Joy, and L. Aleksunis determine the effect of seven nsSNPs on MRP2/ABCC2 trafficking and transport (In Vitro transport activity and trafficking of MRP2/ABCC2 polymorphic variants). On the basis of experimental data and by canvassing large scale genomics databases, L. Li, L. Zhang, P.F. Binkley, W. Sadee, and D. Wang identify a regulatory variant in PRKCA (encoding protein kinase $\mathrm{C} \alpha$ ), with selective effect in the heart and associated with cardiac abnormalities (Regulatory variants modulate protein kinase $\mathrm{C} \alpha$ (PRKCA) gene expression in human heart). Lastly, G. A. Higgins et al. present a novel large-scale data analytics approach to understanding genomic and epigenetic factors in the response to valproate in treatment of traumatic brain injury, a harbinger of novel avenues towards bringing genomics research to clinical fruition (Network reconstruction reveals that valproic acid activates neurogenic transcriptional programs in adult brain following traumatic injury). Taken together, these articles provide a topical cross section highlighting developments in personalized therapeutics and pharmacogenomics.

Implementation of personalized health care is an ardent process, as translation of medical advances into a highly complex, turbulent reality of modern society meets substantial obstacles and is painfully slow. Extending benefits to all layers of human society presents a formidable barrier to improving health care across the world. Thus, we are deeply challenged in an era of disruptive social and technical changes that occur with increasing rapidity. Living up to these expectations, pharmaceutical scientist have a critical role to play in bringing the benefits of personalized health care to fruition.

\section{REFERENCES}

1. Zusi K. Stunning advances over the past 30 years. The Scientist. 2016;10:32-44.

2. Sharp P, Jacks T, Hockfield S. Capitalizing on convergence for health care. Science. 2016;352(6293):1522-3.

3. Denny JC. Translational Bioinformatics: Big data in EHRs, Omics for drugs, and personal Genomics. Nashville: Vanderbilt University Medical Center, IMIA Yearbook of Medical Informatics; 2014. P. 199-205.

4. Fan S, Hansen ME, Lo Y, Tishkoff SA. Going global by adapting local: review of recent human adaptation. Science. 2016;354:54-9.

5. Gluckman P, Beedle A, Hanson M. Principles of evolutionary medicine. Oxford: Oxford Biology; 2009.

6. Azad A, Sadee W, Schlesinger LS. Innate immune gene polymorphisms in tuberculosis. Infect Immun. 2012;80:3343-59. 
7. Phillips KA, Veenstra DL, Oren E, Lee K, Sadee W. The potential role of pharmacogenomics in reducing adverse drug reactions: a systematic review. J Am Med Assoc. 2001;286:2270-9.

8. Oaklander M. The exercise cure time magazine. 2016. Sept.12-19.

9. Kvedar J. The Internet of Healthy Things. Is chronic disease management beyond our reach? 2016. http://theinternetofhealthythings. com/

10. Turek FW. Circadian clocks: not your grandfather's clock. Science. 2016;354:992-3.

11. Personalized Medicine Coalition. Progress Report 17, no 3, May/ June 2016.

12. Ribeil JA, Hacein-Bey-Abina S, Payen E, Magnani A, Semeraro M, Magrin E, Caccavelli L, Neven B, Bourget P, El Nemer W, Bartolucci P, Weber L, Puy H, Meritet JF, Grevent D, Beuzard Y, Chrétien S, Lefebvre T, Ross RW, Negre O, Veres G, Sandler L, Soni S, de Montalembert M, Blanche S, Leboulch P, Cavazzana M. Gene therapy in a patient with sickle cell disease. N Engl J Med. 2017;376:848-55

13. Yin H, Kauffman KJ, Anderson DG. Delivery technologies for genome editing. Nat Rev Drug Discov. 2017.

14. Leslie M. Fighting autoimmunity with immune cells. Science. 2016;353:14.

15. Reardon DA, Wen PY, Wucherpfennig KW, Sampson JH. Immunomodulation for glioblastoma. Curr Opin Neurol. 2017;30(3):361-9.

16. Zaghlool SB, Al-Shafai M, Al Muftah WA, Kumar P, Gieger C, Waldenberger M, Falchi M, Suhre K. Mendelian inheritance of trimodal $\mathrm{CpG}$ methylation sites suggests distal cis-acting genetic effects. BMC Genomics. 2017;18(1):150.

17. Erickson RP. Recent advances in the study of somatic mosaicism and diseases other than cancer. Curr Opin Genet Dev. 2014;26: 73-8.
18. Fuster JJ, MacLauchlan S, Zuriaga MA, Polackal MN, Ostriker AC, Chakraborty R, Wu CL, Sano S, Muralidharan S, Rius C, Vuong J, Jacob S, Muralidhar V, Robertson AA, Cooper MA, Andrés V, Hirschi KK, Martin KA, Walsh K. Clonal hematopoiesis associated with TET2 deficiency accelerates atherosclerosis development in mice. Science. 2017;355:842-7.

19. Rood J. Individualized therapies: from human to pets - and back again. The Scientist. 2016;10:70-2.

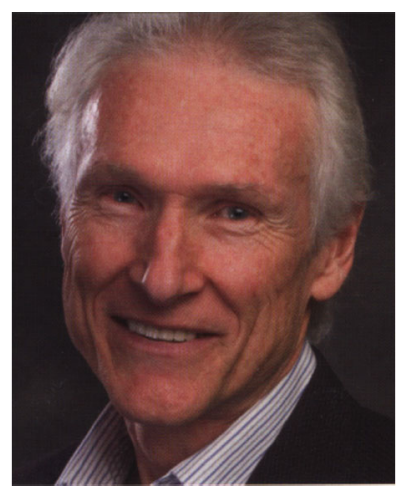

Dr. Wolfgang Sadee is Felts Mercer Professor of Medicine and Pharmacology, in the Department of Cancer Biology and Genetics, College of Medicine, the Ohio State University, Columbus $\mathrm{OH}$, and Director, Center for Pharmacogenomics, with adjunct appointments in Psychiatry, Pharmacy, and Public Health, and member of the Davis Heart \& Lung Research Institute, and OSU Comprehensive Cancer Center. With a doctorate degree in Pharmaceutical Chemistry, FU Berlin, he served on the faculty of USC and UCSF until 2002. Dr. Sadee's research focuses on discovery of genetic variants affecting disease risk and drug response, as biomarkers for individualized therapy. He also has developed candidate drugs for treatment of opioid adverse effects and addiction, and neonatal abstinence syndrome. Dr. Sadee has $\sim 400$ publications and several patents. He is founding editor of Pharmaceutical Research and The AAPS Journal, and has received several awards, e.g., Distinguished Scientist Award from the AAPS. 